\title{
Facilitators and challenges experienced by first-year nursing students at the University of Fort Hare, South Africa, when conducting home visits
}

\author{
N L Gosangaye, ${ }^{1}$ BA Nursing Science; K Mostert ${ }^{2} \mathrm{MBA}, \mathrm{PhD}$ \\ ${ }^{1}$ Department of Nursing Science, Faculty of Health Sciences, University of Fort Hare, East London, South Africa \\ ${ }^{2}$ Department of Physiotherapy, Faculty of Health Sciences, University of Pretoria, South Africa
}

Corresponding author: N L Gosangaye (ngosangaye@ufh.ac.za)

\begin{abstract}
Background. To be socially responsible, tertiary education institutions collaborate with local communities. Community engagement is a teaching and learning strategy that enhances the learning experiences of students while interacting with community members. Assessing a family at home is a curriculum task that strengthens a primary healthcare approach to nursing education.

Objectives. To explore and describe facilitators and challenges experienced by first-year nursing students regarding family assessment during home visits. Methods. An explorative, descriptive, qualitative research approach was used. Four focus group discussions with 6 participants in each group were conducted with purposively selected first-year nursing students. Data were analysed according to Tesch's method.

Results. Participants $(N=24)$ were allocated to the focus group discussions. Facilitators of learning included relevant community and home settings, which provided diversity in learning opportunities. Challenges included absence of a formal orientation to the learning opportunity, language barriers and lack of basic apparatus.

Conclusion. Participants reported facilitators and challenges of family health assessment and health education. The first step should be to build on the facilitators and address the challenges in an action research project.
\end{abstract}

Afr J Health Professions Educ 2020;12(4):169-171. https://doi.org/10.7196/AJHPE.2020.v12i4.1390

Universities have a social responsibility towards society through consultation with communities and by providing a service to individuals, families and groups in geographical areas surrounding the university. Health science faculties, therefore, endeavour to reach excellence in this regard. A requirement of the South African Nursing Council (SANC) is communitybased education, including negotiation of entry into a community, assessing a community's needs and strengths, and providing services, such as consultation in clients' home environments.

A strategy by which to enhance community engagement and primary healthcare is to obtain a clear picture of the health-related strengths and needs of families in a specific community during home visits. ${ }^{[1]}$ Home visits act as an opportunity for students to compile a family health profile and to increase cultural sensitivity, with a simultaneous positive impact on healthcare delivery. ${ }^{[2]}$ Students can practise skills in an environment where they obtain regular feedback and gain exposure to authentic client contexts. Service-learning modules, therefore, require substantial interactions outside the classroom to maintain student engagement. ${ }^{[3]}$ The development of trusting relationships, listening to families' stories and learning about old age take place during home visits. ${ }^{[4]}$ Students reported that they gained confidence and self-esteem. ${ }^{[4]}$

NLG observed that at the institution of higher education where she was involved, first-year students seemed reluctant to interact with the community, which was evident from frequent apologies not to carry out home visits, by remaining in the bus that transported them to the community, and from disappearing into the community.

Therefore, the study aimed to explore and describe facilitators and challenges of student nurses who have to learn how to compile a health- related family profile and provide basic health education, as well as conduct an effective and efficient visit to a client's home as part of the curriculum at a particular institution of higher education in South Africa (SA).

\section{Methods}

The community served by the Department of Nursing Science, University of Fort Hare (UFH), was the setting for the research. All first-year students were expected to compile a health profile (using household survey forms) of families during a home visit, which included completing a health screening questionnaire.

This was an explorative, descriptive and qualitative study. Twenty-four nursing students were purposively selected from the 2017 and 2018 first-year cohorts who did home visits in the same community. Students were recruited from both genders, according to level of seniority (entered university straight from matric or from another course) and different performance levels (grades). They were divided into 4 heterogeneous focus groups ( $n=6$ per group). The discussions were conducted by lecturers, who had Bachelor degrees and experience in conducting such discussions. The opening question at the discussion was: 'What were your experiences during the home visits?' Follow-up questions were: 'What made it easier for you to learn during the home visits?' and 'What challenges did you experience?' More probing questions were asked based on the discussion.

\section{Ethical approval}

The discussions were recorded after participants gave written informed consent (University Research Ethics Committee; ref. no. REC-100118-54). 
Subsequently NLG transcribed the interviews, while both authors independently coded, categorised and identified themes. ${ }^{[5]}$ After each round of analysis, they negotiated consensus.

\section{Results}

The ages of the 24 participants ranged from 19 to 23 years, with the majority being female (70\%) (Table 1 ). Compiling a family profile and providing basic health education were facilitated and enriched by the nature of the community and households that the lecturers selected for home visits. These settings provided an opportunity for participants to learn about diverse conditions and health needs, contextual factors and determinants of health, culture and primary healthcare. Students visited the community in pairs, which added to better learning.

A few factors posed challenges to participants to reach the learning objectives of a home visit, i.e.: insufficient orientation regarding possible challenges that they would encounter; the household survey form being in English (which many of the clients did not understand); insufficient knowledge of common conditions; insufficient consultation skills; lack of basic equipment (e.g. a glucometer); the lecturer often not being available to assist; unrealistic expectations by community members (e.g. receiving groceries); adults and the elderly resisting being questioned and educated by young students; and students doing short once-off visits, which limited the building of rapport with clients.

\section{Discussion}

This study explored and described facilitators and challenges of first-year nursing students at an SA university to complete the learning outcomes of a healthrelated household questionnaire and basic health education during home visits.

\section{Facilitators of learning}

The selected settings offered an opportunity for participants to reach the learning outcomes. Applying basic knowledge about common diseases,

Table 1 . Themes and categories supported by verbatim quotes (one per category)

\begin{tabular}{ll}
\hline Themes & Categories \\
\hline Facilitators of learning & $\begin{array}{l}\text { The community and households offered } \\
\text { opportunities to learn about: } \\
\text { Different conditions and health needs } \\
\text { Different contextual factors and social } \\
\text { determinants of health }\end{array}$ \\
& $\begin{array}{l}\text { Different cultures } \\
\text { Opportunities for primary healthcare activities } \\
\text { The arrangement of the task as an activity in a pair } \\
\text { or group } \\
\text { Insufficient orientation about possible challenges }\end{array}$
\end{tabular}

Student quotes

'We were able to advise clients on the importance of attending child health clinic, basic hygiene, contraceptive methods, prenatal visits, adherence to treatment.'

'We were able to appreciate importance of communication, to understand the influence of contextual factors on health, e.g. overcrowding and understaffed clinics.'

'We were able to appreciate and learn about effect of cultural factors, e.g. access to healthcare via traditional healers and suspicion of clinics.' 'We identified extensive need for health education.'

'This was advantageous because we were able to help and support one another when confronted with a problem.'

'We must be orientated about possible challenges that we will encounter during home visits.'

Language barriers

'Household survey form is written in English. It would be better if survey form is written in the vernacular language for clients to understand the question that was asked during home visits. Household survey form should be written in a language that is best suitable for all people in the community.'

Lack of basic knowledge of common conditions, e.g. tuberculosis and diabetes

Lack of consultation skills

Lack of basic equipment, e.g. glucometer Lecturer often not available to assist with consultation

Unrealistic and non-nursing-related community expectations, e.g. for groceries

Resistance by adults and the elderly to adhere to education provided by health education offered by young students

Once-off visits us to be knowledgeable about disease, e.g. tuberculosis, diabetes. As we were allocated to visit homes early during first semester, we were not yet knowledgeable about certain conditions.'

'As first year during first semester we were not taught about how to conduct consultations.'

'We sometimes did not have the necessary equipment, e.g. a glucometer.' 'We were often confronted with challenges that we could not handle'

'We were not fully orientated about challenges we would meet when doing home visits.'

'It was a very traumatic experience. I felt guilty because I could not help that woman.'

'The facilitator was not always available to company us. As a result the elderly resisted interventions offered by young students.'

'Home visits were few. As a result the opportunity to build necessary rapport was not adequate.' 
determinants of health, culture and primary healthcare enhanced the success of reaching these outcomes. This knowledge is fundamental to the practice of home visits during training. ${ }^{[6]}$ Consolidating knowledge about these factors in the early stage of the educational programme is important. ${ }^{[1,7]}$

\section{Challenges to learning}

Participants were challenged, as they did not receive sufficient orientation for community work. This prevented the building of relationships of trust with community members, commitment to community work and management of activities, even more so when community members were not involved in the orientation. ${ }^{[8]}$ It is self-explanatory that a language barrier between clients and participants and lack of consultation skills would challenge completing the household survey and health education. ${ }^{[9,10]}$ In contrast to short-term services as part of service learning, ${ }^{[11]}$ the participants in the current study did not receive basic equipment, which limited their assessment of clients in terms of blood pressure and blood glucose levels.

Student learning suffers in the absence of a supervisor. Clinical facilitators and lecturers are competent regarding the holistic assessment of patients' physical status and of planning, co-ordinating, implementing and evaluating patient-centred evidence-based care and rehabilitation, which they can share with students. ${ }^{[12,13]}$

Furthermore, unrealistic expectations by community members also hampered participant learning. ${ }^{[1]}$ Students were not accompanied by an older person on the home visits. As the students were mostly younger than the family members, this posed a challenge when completing the household survey and health education owing to a strong hierarchy in Xhosa households, whereby younger adults have to be respectful of older members, as in other collective cultures. ${ }^{[4]}$

\section{Once-off visits}

Inadequate exposure because of the absence of follow-up visits was a hindrance to learning. Without a follow-up visit less trust was built between participants and clients. This inadequate arrangement did not allow for the bonding of the student and the family, which in turn hampered the humanisation of care based on a trusting relationship. ${ }^{[2,6]}$ Compared with studies that can be used for benchmarking, ${ }^{[1,2]}$ working in pairs or groups was not a strategy in the curriculum of the current study.

\section{Study limitations}

A limitation of the study was that the lecturers conducted the interviews, leading to an unbalanced power relationship between them and the participants. However, more challenges than facilitators were reported, suggesting that honest participation was not inhibited.

\section{Conclusion}

Although home visits can provide a valuable learning opportunity at the nursing department at UFH, student participants reported both facilitators and challenges regarding how to carry out a family health assessment and health education. The next step would be to build on the facilitators and address the challenges in an action research project.

\section{Declaration. None.}

Acknowledgements. The authors are sincerely grateful to Dr M Mammen, lecturer, and Mrs NB Qomfo, clinical facilitator, Department of Nursing Science, UFH, who acted as critical academic friends during the proposal development, and Mrs SM Mnwana, who assisted with conducting the focus group discussions. We also thank the students who participated in the focus group discussions.

Author contributions. NLG posed the research question, collected and analysed the data and wrote the first draft of the manuscript. KM guided the development of the proposal, oversaw the data collection, analysed the data and critically read and improved the manuscript.

Funding. The sub-Saharan African FAIMER Regional Institute (SAFRI) and the Department of Nursing Science, Faculty of Health Sciences, UFH, funded this study. Conflicts of interest. None.

\footnotetext{
1. Zomorodi M, Odom T, Askew NC, Leonard CR, Sanders KA, Thompson D. Hotspotting. Nurs Educ 2018;43(5):247-250. https://doi.org/10.1097/nne.0000000000000523

2. Uwitonze A. An exploration of nursing students' experiences of a community-based health promotion and illness prevention programme in a selected school of nursing in KwaZulu-Natal. PhD thesis. Durban: University of KwaZulu-Natal, 2017

3. Ngui KS, Voon ML, Lee MH. Integrating community engagement with management education. Educ Train 2017;59(6):579-589. https://doi.org/10.1108/et-04-2016-0078

4. Walton J, Blossom $\mathrm{H}$. The experience of nursing students visiting older adults living in rural communities. J Prof Nurs 2013;29(4):240-251. https://doi.org/10.1016/j.profnurs.2019.05.010 8

5. Creswell J. Research Design: Qualitative, Quantitative, and Mixed Methods Approaches. Thousand Oaks, CA SAGE, 2018

SAGE, 2018.
6. Borges FR, Avelino CCV, da Costa LCS, Lourenço DS, de Sá MD, Goyatá SLT. Teaching about home visits to university students. Revista da Rede de Enfermagem do Nordeste 2017;18(1):129-138. https://do org/10.15253/2175-6783.2017000100018

7. Iwasaki R, Hirai K, Kageyama T, et al. Supporting elder persons in rural Japanese communities through preventive home visits by nursing students: A qualitative descriptive analysis of students' reports. Public Health Nurs 2019;36(4):557-563. https://doi.org/10.1111/phn.12596

8. Tanabe S, Yanagisawa S, Waqa-Ledua S, Tukana M. Identifying characteristic features of community orientatio among community health nurses in Fiji. Nursing Open 2019;6:1113-1123. https://doi.org/10.1002/nop2.305

9. Czop Assaf L, Lussier KO. Dream camp: Drawing on community cultural wealth capital to make sense of career dreams. Language Culture Curriculum 2020;33(1):84-99. https://doi.org/10.1080/07908318.2019.1569020

10. Hwang WJ, Kim JA. Development and evaluation of a home-visit simulation scenario for elderly people with diabetes mellitus who live alone. J Comm Health Nurs 2020;37(2):89-102. https://doi.org/10.1080/07370016.2 020.1736399

11. O'Handley H, Erlinger A. Recommendations for planning short-term nursing student mission trips. J Profes Nurs 2019;35(4):329-334. https://doi.org/10.1016/j.profnurs.2019.01.006

12. Vaartio-Rajalin H, Näsman Y, Fagerström L. Nurses' activities and time management during home healthcare visits. Scand J Caring Sci 2019. https://doi.org/10.1111/scs.12813

13. Moffatt FW, White FM, Sheffer C. A simulated postpartum home visiting program with undergraduate nursing students, faculty, and actors. J Obstet Gynecol Neonat Nurs 2017;46(3):S16.
}

Accepted 15 September 2020 